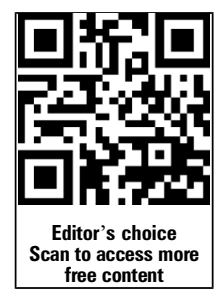

\title{
An estimate of the proportion of symptomatic gonococcal, chlamydial and non-gonococcal non- chlamydial urethritis attributable to oral sex among men who have sex with men: a case-control study
}

\author{
Lindley A Barbee, ${ }^{1,2}$ Christine M Khosropour, ${ }^{3}$ Julia C Dombrowski, ${ }^{1,2}$ \\ Lisa E Manhart, ${ }^{3,4}$ Matthew R Golden 1,2,3
}

${ }^{1}$ Department of Medicine, Division of Allergy and Infectious Diseases, University of Washington, Seattle, Washington, USA

${ }^{2}$ Public Health, Seattle \& King County HIV/STD Program ${ }^{3}$ Department of Epidemiology, University of Washington, Seattle, Washington, USA ${ }^{4}$ Department of Global Health, University of Washington, Seattle, Washington, USA

\section{Correspondence to} Dr Lindley A Barbee, Department of Medicine, Division of Allergy and Infectious Disease, University of Washington, 325 9th Ave, Box 359777 Seattle, WA 98104, USA; lbarbee@u.washington.edu

Received 15 June 2015 Revised 29 July 2015 Accepted 4 August 2015 Published Online First 21 August 2015

\section{CrossMark}

\section{To cite: Barbee $L A$,} Khosropour CM

Dombrowski JC, et al. Sex Transm Infect 2016;92 155-160.

\section{ABSTRACT \\ Background Sexually transmitted infections (STIS) of the pharynx are common among men who have sex with men (MSM); the degree to which these infections are transmitted through oral sex is unknown. \\ Methods We conducted a case-control study of MSM attending Public Health—Seattle \& King County STD Clinic between 2001 and 2013 to estimate the proportion of symptomatic urethritis cases attributable to oral sex using two methods. First, we categorised men into the following mutually exclusive behavioural categories based on their self-reported sexual history in the previous 60 days: (1) only received oral sex (IOS); (2) $100 \%$ condom usage with insertive anal sex plus oral sex (PIAI); (3) inconsistent condom usage with anal sex (UIAI); and (4) no sex. We then determined the proportion of cases in which men reported the oropharynx as their only urethral exposure (IOS and PIAI). Second, we calculated the population attributable risk per cent (PAR\%) associated with oral sex using Mantel-Haenszel OR estimates.}

Results Based on our behavioural categorisation method, men reported the oropharynx as their only urethral exposure in the past 60 days in $27.5 \%$ of gonococcal urethritis, $31.4 \%$ of chlamydial urethritis and $35.9 \%$ non-gonococcal, non-chlamydial urethritis (NGNCU) cases. The PAR\%s for symptomatic gonococcal urethritis, chlamydial urethritis and NGNCU attributed to oropharyngeal exposure were $33.8 \%, 2.7 \%$ and $27.1 \%$, respectively.

Conclusions The pharynx is an important source of gonococcal transmission, and may be important in the transmission of chlamydia and other, unidentified pathogens that cause urethritis. Efforts to increase pharyngeal gonorrhoea screening among MSM could diminish STI transmission.

\section{INTRODUCTION}

Bacterial sexually transmitted infections (STIs), and gonorrhoea and chlamydia in particular, are common among men who have sex with men (MSM). In 2012, at 12 sentinel surveillance sites across the USA, the median proportion of MSM diagnosed with gonorrhoea and chlamydia was $16.4 \%$ and $12 \%$, respectively. ${ }^{1}$ The majority of gonococcal and chlamydial infections in this population occur at extragenital sites, that is, the oropharynx and/or the rectum. ${ }^{1}$ Yet, these infections are usually asymptomatic ${ }^{2}$ and often underdiagnosed due to inadequate screening. ${ }^{1}$ As common, asymptomatic and underdiagnosed infections, gonorrhoea and chlamydia of the oropharynx may play an important role in sustaining the extremely high rates of bacterial STI observed in this population. However, the extent to which oral sex is responsible for transmitting Neisseria gonorrhoeae, Chlamydia trachomatis and other causes of urethritis is unknown. In this study, we estimate the proportion of symptomatic urethritis among MSM attributable to oral sex using two methods: (1) using reported sexual behaviours, we determined the proportion of cases of symptomatic urethritis among men whose only reported exposure was the oropharynx, and (2) we calculated the population attributable risk per cent (PAR\%). As a secondary analysis, we compare asymptomatic urethral infections to cases of symptomatic urethritis.

\section{METHODS}

We conducted a case-control study of all new problem clinic visits by MSM at the Public Health - Seattle \& King County (PHSKC) STD Clinic between 1 January 2001 and 31 December 2013. Cases were defined as MSM visits where symptomatic urethritis was diagnosed, and controls were all MSM visits when no urethral infection was diagnosed. Men may have been diagnosed with urethritis on more than one visit over the 13-year study period. Each new episode of urethritis was counted separately. New problem visits were defined as visits that occurred at least 30 days after the last clinic visit, or a visit for a new complaint within 30 days of a prior clinic visit. All data were collected as part of routine medical care, recorded in the clinic's electronic database, and deidentified for analysis.

\section{Clinical and laboratory procedures, and}

definitions of outcomes and behavioural risks

Clinicians record exam findings for all patients seen in the STD Clinic using a standardised reporting form. Sexual histories were also collected by clinicians on standardised forms until 2010 when the clinic initiated a computer-assisted self-interview for the collection of sexual behaviour data. Clinicians collect specimens for anatomical sitespecific STI testing based on patients' sexual 
history, symptoms and physical exam findings. For this study, we focused on symptomatic urethritis because most cases of symptomatic urethritis result from exposures in the prior 60 days, ${ }^{3} 4$ the period for which our clinic collects sexual history data related to urethritis. We defined symptomatic urethritis, our cases, using the following criteria: (1) patient complaint of urethral discharge, dysuria or other urethral discomfort, and (2) $\geq$ five leucocytes per high power field (HPF) in three fields on a Gram stain of a urethral discharge. Controls were defined as men who attended the clinic during the study period who had no urethral sign or symptoms and were not diagnosed with urethral infection on screening tests. Men who presented with urethral symptoms but did not have $\geq 5$ leucocytes/HPF were excluded from analyses.

Urethral gonorrhoea was diagnosed using culture until 2002, and a combination of culture and urine or urethral nucleic acid amplification tests (NAATs) thereafter. Our clinic protocol since 2002 has been to collect urine for NAATs from symptomatic and asymptomatic MSM and to collect culture if discharge was apparent on clinical exam. We employed the Aptima Combo 2 (Hologic, San Diego, California, USA) for gonorrhoea and chlamydial NAAT testing throughout the study period. Chlamydial urethritis was diagnosed by culture prior to 2002 and by NAAT thereafter. Symptomatic non-gonococcal, non-chlamydial urethritis (NGNCU; urethritis without an identifiable pathogen) was diagnosed if men met our criteria of symptomatic urethritis and had negative microbiological testing for C. trachomatis and N. gonorrhoeae.

We also examined asymptomatic urethral infections among all MSM attending the PHSKC STD Clinic during the study period for comparison with symptomatic urethritis. Asymptomatic urethral infections were defined as (1) positive NAAT or culture for gonorrhoea or chlamydia; or, $\geq 5$ leucocytes per HPF in three fields on a Gram stain of urethral swab; and (2) the absence of patient-reported urethral symptoms. We defined persons as having asymptomatic NGNCU if they met our criteria for having asymptomatic urethral infection and had negative laboratory testing for gonorrhoea and chlamydia. Asymptomatic screening for urethral gonorrhoea and chlamydia occurred by culture from 2001-2002 and by NAAT after 2002.

We defined any man who reported sex with another man in the previous 12 months as being an MSM. If data on gender of sex partner were missing and a study subject had a prior clinic visit, we defined men as MSM based on information from their most recent prior clinic visit in which the medical record included data on gender of sex partners.

\section{Statistical analysis}

We used two separate methods to estimate the percentage of cases of gonococcal urethritis, chlamydial urethritis and NGNCU attributable to oral sexual exposure. Our first method - the behavioural categorisation approach-involved creating four mutually exclusive categories of urethral exposure based on men's reported behaviour in the 60 days prior to their clinic visit: (1) MSM who reported only insertive oral sex with another man (penile-oral exposure) (insertive oral sex, IOS); (2) men who reported only protected ( $100 \%$ condom use) insertive anal sex plus oral sex with men (protected insertive anal sex, PIAI); (3) men who reported no or inconsistent condom use with insertive anal sex with or without oral sex (unprotected insertive anal sex, UIAI); and (4) MSM who reported no sex in the prior 60 days (none). We excluded men from the study for whom we did not have complete data on sexual activity and/or condom use $(\mathrm{N}=6606,16.5 \%)$. Using the above behavioural categories, we calculated the percentage of cases of urethritis or urethral infections stratified by aetiology for each urethral exposure category. Here we assumed that men with symptomatic urethritis who reported receiving fellatio but no unprotected anal sex (IOS and PIAI groups) were infected through exposure to a sex partner's oropharynx and estimated the proportion of cases attributable to oral sex. Because men in the UIAI group may also have acquired symptomatic urethritis from oral sex, our mutually exclusive sexual behaviour categories could underestimate the risk of symptomatic urethritis associated with oral sex; insofar as sexual histories were inaccurate or did not include the period of STI acquisition, they may also be inaccurate.

Our second approach for estimating the proportion of urethritis cases attributable to oral sex involved calculating the PAR\%. To do this, we calculated the Mantel-Haenszel OR for the association between each different sexual behaviour (receptive oral sex, protected insertive anal intercourse, unprotected insertive anal sex, vaginal sex, and the absence of any sexual behaviour) and gonococcal urethritis, chlamydial urethritis and NGNCU, removing the restriction of mutual exclusivity. That is, we created four models for each of the three aetiologies of urethritis, one for each of the sexual behaviours while including the other sexual behaviours in the model. This method gave us a summary OR for the association of a given sexual behaviour and aetiology across sexual behaviour strata. Because the prevalence of our outcomes (aetiology-specific urethritis) was less than $10 \%$, we assumed that the OR approximated the relative risk (RR) and used these estimates to calculate the PAR\% using the following formula:

$$
\mathrm{P}_{\mathrm{c}} \times[(\mathrm{RR}-1) / \mathrm{RR}] \times 100
$$

where $P_{c}$ is the proportion of cases exposed (ie, the prevalence of the sexual behaviour among the cases). ${ }^{5}$ To compare descriptive statistics of our case and control populations, we used $\chi^{2}$ tests for categorical variables and $t$ tests for continuous variables. All analyses were conducted using Stata V.12.0 (StataCorp, College Station, Texas, USA) and an $\alpha$ of 0.05 was considered statistically significant.

\section{RESULTS}

\section{Study population}

Between 1 January 2001 and 31 December 2013, there were 40078 new problem visits by MSM at the PHSKC STD Clinic, of which 33472 had complete sexual behaviour data and were included in the analysis. MSM complained of urethral symptoms at 6655 visits, and clinicians diagnosed symptomatic urethritis during 5277 visits (table 1). Overall, men with symptomatic urethritis were not different than the general MSM population attending the PHSKC STD Clinic in terms of age, race and number of sexual partners. Men with symptomatic urethritis were more likely to report being HIV-positive than controls, and the distribution of sexual behaviours was markedly different for the men with symptomatic urethritis compared with controls and to the overall clinic population of MSM, with more men with symptomatic urethritis reporting UIAI and fewer reporting only oral sex or PIAI.

\section{Aetiology of symptomatic urethritis and asymptomatic urethral infections}

Clinicians diagnosed a total of 6464 urethral infections during the study period, the majority of which were symptomatic 
Table 1 Study population, demographics and sexual behaviours among men who have sex with men (MSM) attending Public Health-Seattle \& King County (PHSKC) STD Clinic (N=33 472) between 1 January 2001 and 31 December 2013

\begin{tabular}{|c|c|c|c|c|}
\hline & $\begin{array}{l}\text { Total study } \\
\text { population* } \\
\mathrm{N}=33472 \dagger\end{array}$ & $\begin{array}{l}\text { Controls: no urethral } \\
\text { infection } \\
\mathrm{N}=25705 \\
(76.8 \%)\end{array}$ & $\begin{array}{l}\text { Cases: symptomatic } \\
\text { urethritis } \\
n=5277 \\
(15.8 \%)\end{array}$ & $\begin{array}{l}\text { Asymptomatic } \\
\text { urethritis } \\
n=1112 \\
(3.3 \%)\end{array}$ \\
\hline Patient reported urethral symptoms $(\mathrm{N}, \%)$ & $6655(19.9)$ & & $5277(100)$ & \\
\hline Age (mean, SD) & $35.5(10.9)$ & $35.6(11.2)$ & $34.9(9.7)$ & $33.7(10.4)$ \\
\hline \multicolumn{5}{|l|}{ Race $(\mathrm{N}, \%)$} \\
\hline White & $23370(73.0)$ & $17847(69.4)$ & $3771(74.8)$ & $765(68.8)$ \\
\hline Black & $2457(7.7)$ & $1747(6.8)$ & $492(9.8)$ & $94(8.5)$ \\
\hline Hispanic & $1935(6.1)$ & $1561(6.1)$ & $250(5.0)$ & $74(6.7)$ \\
\hline Asian & $1938(6.1)$ & $1635(6.4)$ & $207(4.1)$ & $51(4.6)$ \\
\hline Native American/Alaskan native & $390(1.2)$ & $300(1.2)$ & $54(1.1)$ & $17(1.5)$ \\
\hline Other & $1907(6.0)$ & $1485(5.8)$ & $268(5.3)$ & $65(5.8)$ \\
\hline $\begin{array}{l}\text { Number of sex partners in the past } 2 \text { months } \\
\text { (median, IQR) }\end{array}$ & $3.7(3)$ & $3.8(3)$ & $3.3(3)$ & $3.5(3)$ \\
\hline \multicolumn{5}{|l|}{ Sexual behaviours in the past 2 months $(\mathrm{N}, \%)$} \\
\hline Only oral sex (IOS) & $5131(15.3)$ & $4148(16.1)$ & $639(12.1)$ & $108(9.7)$ \\
\hline $\begin{array}{l}\text { Protected insertive anal intercourse and oral sex } \\
\text { (PIAI) }\end{array}$ & $7691(23.0)$ & $6106(23.8)$ & $1065(20.2)$ & $210(18.9)$ \\
\hline Unprotected insertive anal intercourse (UIAI) & $19898(59.5)$ & $14817(57.6)$ & $3501(66.3)$ & $777(69.9)$ \\
\hline Vaginal sex & $412(1.2)$ & $355(1.4)$ & $29(0.6)$ & $12(1.1)$ \\
\hline None & $340(1.0)$ & $279(1.1)$ & $43(0.8)$ & $5(0.4)$ \\
\hline HIV-positive (N, \%) & $3923(11.7)$ & $2738(10.7)$ & $870(16.5)$ & $149(13.4)$ \\
\hline \multicolumn{5}{|l|}{ Urethral infections by aetiology } \\
\hline Gonorrhoea & $1614(4.8)$ & & 1509 (28.6) & $95(8.5)$ \\
\hline Chlamydia & $1119(3.3)$ & & 599 (11.4) & $455(40.9)$ \\
\hline $\begin{array}{l}\text { Non-gonococcal non-chlamydial urethritis } \\
\text { (NGNCU) }\end{array}$ & $3482(10.4)$ & & $2935(55.6)$ & $547(49.2)$ \\
\hline Gonorrhoea and chlamydia & $249(0.7)$ & & $234(4.4)$ & $15(1.4)$ \\
\hline
\end{tabular}

${ }^{*}$ MSM new problem visits with complete data to permit categorisation based on sexual behaviours.

tIncludes $1378(4.1 \%)$ visits in which men complained of urethral symptoms but were not diagnosed with urethritis by our definition. This included 75 with positive tests for gonorrhoea and/or chlamydia. These 1378 are excluded from the case-control and asymptomatic infection analyses.

¥Percentages do not add to $100 \%$ due to non-response on some items.

$(\mathrm{n}=5277)$ (table 1). Gonococcal infections and NGNCU were primarily symptomatic ( $94 \%$ and $84 \%$, respectively), whereas, only $61 \%$ of chlamydial infections were symptomatic. Of the asymptomatic infections, 150 (13.1\%) were evaluated as known sexual contacts to men with gonorrhoea or chlamydia. There was no appreciable difference in the distribution of sexual behaviours or the number of sexual partners in the previous

Table 2 Number and percentage of symptomatic cases of gonococcal, chlamydial and non-gonococcal, non-chlamydial urethritis (NGNCU) occurring in men who have sex with men (MSM) STD clinic patients by sexual exposures in the prior 60 days

\begin{tabular}{lccc}
\hline $\begin{array}{l}\text { Presumed anatomical source } \\
\text { of infection based on sexual } \\
\text { behaviour }\end{array}$ & Gonorrhoea* $^{*}$ & Chlamydia* $^{*}$ & NGNCU \\
\hline Oropharynx & $414(27.5)$ & $188(31.4)$ & $1053(35.9)$ \\
IOS & $129(8.6)$ & $54(9.0)$ & $441(15.0)$ \\
PIAI & $285(18.9)$ & $134(22.4)$ & $612(20.9)$ \\
Rectum (UIAI) & $1088(72.1)$ & $406(67.8)$ & $1824(62.2)$ \\
Vagina & $3(0.2)$ & $3(0.5)$ & $23(0.8)$ \\
None & $4(0.3)$ & $2(0.3)$ & $35(1.2)$ \\
\hline
\end{tabular}

${ }^{*}$ Gonococcal/chlamydial co-infections excluded from this analysis.

IOS, insertive oral sex only; PIAI, protected insertive anal intercourse plus oral sex; UIAI, unprotected insertive anal intercourse.
2 months between the cases of symptomatic urethritis and asymptomatic urethral infections. However, men with symptomatic urethritis were more likely to be infected with HIV than men with asymptomatic infection $(p=0.01)$.

\section{Symptomatic urethritis: behavioural categorisation and population attributable risk}

Table 2 presents the proportion of cases of gonococcal urethritis, chlamydial urethritis and NGNCU occurring in men within each mutually exclusive sexual behavioural category. The oropharynx was reported as the only urethral exposure in the previous 60 days in $27.5 \%$ of symptomatic gonococcal urethritis cases, $31.4 \%$ of symptomatic chlamydial urethritis cases and $35.9 \%$ symptomatic NGNCU cases.

Table 3 presents data on the association of insertive oral sex, protected insertive anal sex, unprotected insertive anal sex, and vaginal sex with the different aetiologies of symptomatic urethritis and the corresponding PAR\%. The PAR\% estimates for symptomatic cases of gonococcal urethritis and NGNCU attributable to transmission through oral sex $(33.8 \%$ and $27.1 \%$, respectively) were roughly similar to estimates derived from our behavioural categorisation. In contrast, the two analytical approaches yielded widely divergent estimates of percentage of cases of symptomatic chlamydial urethritis attributable to oral sex; the calculated PAR\% for chlamydial urethritis associated with oral sex was only $2.7 \%$ whereas the proportion of men 


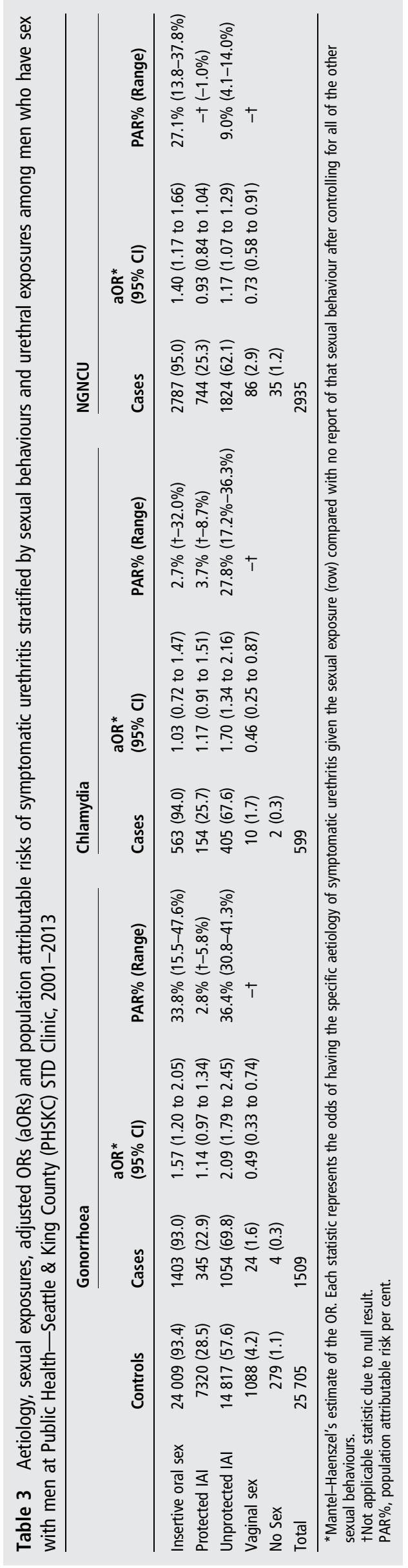

with symptomatic chlamydial urethritis who report only oropharyngeal exposure was over $30 \%$.

Because nearly $40 \%$ of urethral chlamydial infections were asymptomatic, we conducted a secondary analysis exploring the relative risks and $\mathrm{PAR} \%$ for all urethral chlamydial infections associated with oral sex. Although the proportion of symptomatic chlamydial urethritis attributable to oral sex was only $2.7 \%$ (OR 1.03 , 95\% CI 0.72 to 1.47 ), when we included asymptomatic chlamydial infections in the model, oral sex accounted for $13.8 \%$ (PAR\% range, NA-33.3\%) of chlamydial urethral infection.

\section{DISCUSSION}

Our findings suggest that a quarter to a third of all cases of symptomatic gonococcal urethritis and NGNCU among MSM attending an urban STD Clinic can be attributed to oral sex. This conclusion is supported by substantially concordant estimates derived through two separate methods. In contrast to our findings related to gonorrhoea and NGNCU, our two estimation methods yielded widely variable results related to the percentage of cases of chlamydial urethritis attributable to transmission through oral sex. These results strongly support the conclusion that the oropharynx is an epidemiologically important reservoir for gonorrhoea that may play a critical role in sustaining the very high levels of STI transmission observed among MSM.

Our results are largely consistent with prior work suggesting that transmission of STIs from the oropharynx occurs and is important. Bernstein and colleagues in San Francisco previously observed that the risk of urethral chlamydia and gonorrhoea was similar among MSM attending an STD clinic who reported only receiving oral sex and those who reported insertive anal sex, ${ }^{6}$ also suggesting an important role for oral sexual exposures in bacterial urethritis. Similarly, Lafferty and colleagues, reporting data from our STD clinic in Seattle 1993-1994, observed that the risk of urethral gonorrhoea and chlamydia associated with insertive anal and oral sex was similar, and that oral sex was associated with a higher risk of NGNCU than anal sex, ${ }^{7}$ a finding that is consistent with the results of our study. A recent publication from Australia also found no difference in the risk of urethral gonorrhoea between those who reported insertive anal sex and those who denied insertive anal sex. Importantly, using a 12 month time period, that study found that sexual activities other than insertive anal sex did not confer a risk of chlamydial urethritis. ${ }^{8}$ Our findings, which largely agree with these earlier reports, expand on prior observations by providing an estimate of the importance of oral sex in the transmission of STIs and, in particular, demonstrating that a sizeable proportion of gonococcal urethritis in MSM is likely transmitted through oral sex.

Interestingly, a quarter or more of NGNCU in our study was likely acquired through oropharyngeal exposure. The existing literature on the association of non-gonococcal urethritis with oral sex has been mixed. Some investigators have found no association, ${ }^{9-11}$ while others have found an increased risk. ${ }^{12}{ }^{13}$ Bradshaw and colleagues found that oral sex was specifically associated with viral aetiologies (adenovirus and HSV-1), and with urethritis without an identifiable pathogen $(66 \%$ of cases). ${ }^{13}$ Further investigation, specifically to define the organisms responsible for NGNCU associated with oral sex, whether those organisms are distinct from those transmitted via anal or vaginal sex, and the extent to which these infections result in important sequelae is warranted.

The stark discrepancy of the results from the behavioural categorisation analysis and the PAR\% estimates for symptomatic chlamydial infection is noteworthy. Although over 30\% of men 
with symptomatic chlamydial urethritis reported the oropharynx as their only urethral exposure in the past 60 days, the PAR $\%$ was only $2.7 \%$. There are several potential explanations for this difference. First, some patients may have acquired their urethral chlamydial infections prior to the 60-day period for which we collected behavioural data. In an effort to diminish this impact of older infections on our estimates, we focused our main analysis on men with symptomatic urethritis-a group we believed would primarily contain men with recently acquired infections. However, the natural history of chlamydial urethritis is not well defined, and it may be that some men develop symptoms or seek evaluation for symptoms only after a relatively long period of infection. Our divergent results could also reflect inaccuracies in our patients' sexual histories. However, these inaccuracies did not seem to have a large effect on our estimates of the proportion of gonococcal urethritis or NGNCU attributable to oral sex, and it seems implausible that inaccuracies in sexual history world preferentially occur among men with chlamydial infection.

Other sources of data support the idea that gonorrhoea is much more frequently transmitted through oral sex than chlamydial infection. Studies using NAAT to test for pharyngeal gonorrhoea and chlamydia have typically observed relatively low levels of chlamydial positivity $(1.4-2.9 \%),{ }^{2}{ }^{14}$ while the prevalence of pharyngeal gonorrhoea has been documented to be as high as 9.2\%. ${ }^{2}$ The difference between the PAR\% of symptomatic gonococcal and chlamydial urethritis due to oral sex $(33.8 \%$ vs $2.7 \%$, respectively) are consistent with the finding that pharyngeal gonorrhoea is common and pharyngeal chlamydial infection is relatively rare.

Our study is subject to several limitations. First, the study population was composed of MSM attending an urban STD Clinic in the Pacific North-West of the USA and our results may not be generalisable to other populations, particularly heterosexuals, with different sexual repertoires. Second, we used a 60-day time frame to define patients' sexual exposures. As discussed above, if infections were acquired more than 60 days prior to clinic attendance, we may have misclassified patients' exposures and perhaps overestimated the percentage of cases attributable to oral sex using our behavioural categorisation approach. Third, our data on sexual risk behaviours is based on self-report which are subject to recall and social desirability bias. This may also have led us to overestimate the proportion of infections attributable to oral sex if men overestimated their use of condoms. Fourth, our behavioural categorisation estimate of the proportion of urethritis cases transmitted via oral sex assumes that condoms are $100 \%$ effective in preventing gonorrhoea and chlamydial infection transmitted through anal sex, and that all cases of urethritis occurring in MSM who had oral sex and unprotected insertive anal sex were transmitted via anal sex. The first of these assumptions could lead to an overestimate of the percentage of urethritis cases attributable to oral sex, while the second could lead to an underestimate. However, our analysis of the calculated PAR\% does not make this assumption. Additionally, because our Mantel-Haenszel OR calculations used non-mutually exclusive sexual behaviour exposures, there may be some degree of adjustment that attenuates the association between sexual exposure and the risk of symptomatic urethritis. Consequently, our estimates may underestimate the risk of symptomatic urethritis associated with oropharyngeal exposure. Lastly, because we relied on clinic-based data, we were unable to identify cases of NGNCU caused by pathogens such as Mycoplasma genitalium, herpes viruses and adenovirus, which can cause NGNCU, but which are not tested for routinely in our clinic. Although this is a limitation to our study, M. genitalium has not been detected in the oropharynx previously, ${ }^{15}$ and our findings support the idea that oral sex is a likely source of infection for many cases of NGNCU.

In conclusion, we found that a quarter to a third of all cases of symptomatic gonococcal urethritis and NGNCU are likely acquired through oral sex. This observation strongly supports the idea that the failure to identify and eradicate N. gonorrhoeae from the oropharynx plays a critical role in sustaining the extremely high rates of gonorrhoea observed in MSM, and that as yet unidentified oropharyngeal flora may cause a significant proportion of cases of NGNCU. These findings should add new momentum to efforts to promote widespread pharyngeal screening for gonorrhoea among MSM.

\section{Key messages}

- Oral sex accounts for up to a third of all cases of gonococcal and non-gonococcal, non-chlamydia urethritis among men who have sex with men (MSM).

- Chlamydia is also transmitted from the oropharynx to the urethra, though the degree to which this occurs is less certain.

- We should encourage efforts to increase routine screening of the pharynx for gonorrhoea in MSM.

\section{Handling editor Jackie A Cassell}

Acknowledgements The authors thank Dr Noel Weiss for epidemiology methods guidance.

Contributors MRG and LAB conceptualised the study. All authors were involved with the development of the study design. $L A B$ and CMK conducted statistical analyses. $L A B$ drafted the manuscript and, MRG, LAB, CMK, LEM and JCD provided critical revisions of the manuscript.

Funding This work was supported by Public Health-Seattle \& King County, the National Institutes of Health (NIH; Grant Nos. T32 Al07140 to CMK and LAB, K23Al11523791 to LAB and K23MH090923 to JCD), and the University of Washington Center for AIDS Research, an NIH-funded programme (Grant No. P30 Al027757), which is supported by the following NIH institutes and centres: National Institute of Allergy and Infectious Diseases; National Career Institute; National Institutes of Mental Health; National Institute on Drug Abuse; National Institute of Child Health and Human Development; National Heart, Lung, and Blood Institute; and National Institute on Aging.

Competing interests MRG has received research support from Cempra Pharmaceuticals. LEM has served on scientific advisory boards for Qiagen and Hologic, and received research support from Hologic.

Ethics approval University of Washington IRB.

Provenance and peer review Not commissioned; externally peer reviewed.

Presentations This work was presented in part at the 2013 STI and AIDS World Congress in Vienna, Austria on 15 July 2013.

\section{REFERENCES}

1 CDC. Sexually transmitted diseases surveillance, 2012. Atlanta, GA: US Department of Health and Human Services, 2013.

2 Kent CK, Chaw JK, Wong W, et al. Prevalence of rectal, urethral, and pharyngeal chlamydia and gonorrhea detected in 2 clinical settings among men who have sex with men: San Francisco, California, 2003. Clin Infect Dis 2005;41: 67-74.

3 McCutchan JA. Epidemiology of venereal urethritis: comparison of gonorrhea and nongonococcal urethritis. Rev Infect Dis 1984;6:669-88.

4 Boyd JT, Csonka GW, Oates JK. Epidemiology of non-specific urethritis. Br J Vener Dis 1958:34:40-3.

5 Rockhill B, Newman B, Weinberg C. Use and misuse of population attributable fractions. Am J Public Health 1998;88:15-19. 


\section{Epidemiology}

6 Bernstein KT, Stephens SC, Barry PM, et al. Chlamydia trachomatis and Neisseria gonorrhoeae transmission from the oropharynx to the urethra among men who have sex with men. Clin Infect Dis 2009;49:1793-7.

7 Lafferty WE, Hughes JP, Handsfield HH. Sexually transmitted diseases in men who have sex with men. Acquisition of gonorrhea and nongonococcal urethritis by fellatio and implications for STD/HIV prevention. Sex Transm Dis 1997;24:272-8.

8 Nash JL, Hocking JS, Read TR, et al. Contribution of sexual practices (other than anal sex) to bacterial sexually transmitted infection transmission in men who have sex with men: a cross-sectional analysis using electronic health records. Sex Transm Infect 2014;90:55-7.

9 Manhart LE, Gillespie CW, Lowens MS, et al. Standard treatment regimens for nongonococcal urethritis have similar but declining cure rates: a randomized controlled trial. Clin Infect Dis 2013;56:934-42.

10 Totten PA, Schwartz MA, Sjöström KE, et al. Association of Mycoplasma genitalium with nongonococcal urethritis in heterosexual men. J Infect Dis 2001;183:269-76.
11 Wetmore CM, Manhart LE, Lowens MS, et al. Demographic, behavioral, and clinical characteristics of men with nongonococcal urethritis differ by etiology: a case-comparison study. Sex Transm Dis 2011;38:180-6.

12 Gillespie CW, Manhart LE, Lowens MS, et al. Asymptomatic urethritis is common and is associated with characteristics that suggest sexually transmitted etiology. Sex Transm Dis 2013:40:271-4.

13 Bradshaw CS, Tabrizi SN, Read TR, et al. Etiologies of nongonococcal urethritis: bacteria, viruses, and the association with orogenital exposure. J Infect Dis 2006;193:336-45.

14 Patton ME, Kidd S, Llata E, et al. Extragenital gonorrhea and chlamydia testing and infection among men who have sex with men--STD Surveillance Network, United States, 2010-2012. Clin Infect Dis 2014;58:1564-70.

15 Deguchi T, Yasuda M, Yokoi S, et al. Failure to detect Mycoplasma genitalium in the pharynges of female sex workers in Japan. J Infect Chemother 2009;15:410-13. 